\title{
Budding yeast Saccharomyces cerevisiae as a model to study oxidative modification of proteins in eukaryotes ${ }^{\star}$
}

\author{
Volodymyr I. Lushchak ${ }^{\bowtie}$ \\ Department of Biochemistry, Vassyl Stefanyk Precarpathian National University, Ivano-Frankivsk, Ukraine;

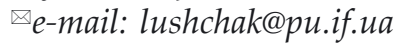

Received: 06 June, 2006; revised: 08 August, 2006; accepted: 31 August, 2006 available on-line: 26 October, 2006

\begin{abstract}
The budding yeast Saccharomyces cerevisiae is a well studied unicellular eukaryotic organism the genome of which has been sequenced. The use of yeast in many commercial systems makes its investigation important not only from basic, but also from practical point of view. Yeast may be grown under both aerobic and anaerobic conditions. The investigation of the response of eukaryotes to different kinds of stresses was pioneered owing to yeast and here we focus mainly on the so-called oxidative stress. It is a result of an imbalance between the formation and decomposition of reactive oxygen species increasing their steady-state concentration. Reactive oxygen species may attack any cellular component. In the present review oxidation of proteins in S. cerevisiae is analyzed. There are two connected approaches to study oxidative protein modification - characterization of the overall process and identification of individual oxidized proteins. Because all aerobic organisms possess special systems which defend them against reactive oxygen species, the involvement of so-called antioxidant enzymes, particularly superoxide dismutase and catalase, in the protection of proteins is also analyzed.
\end{abstract}

Keywords: Saccharomyces cerevisiae, reactive oxygen species, oxidative stress, protein oxidation

\section{INTRODUCTION}

Saccharomyces cerevisiae is probably the best studied eukaryotic organism. This is because yeast is easily grown in laboratory and is an extremely favorable organism for studies of genetic phenomena. $S$. cerevisiae is the common baker's and brewer's yeast and is not only a useful model system, but also an important organism of commerce. Therefore, studies of its biology, physiology, biochemistry and genetics can be expected to have a practical significance. Because yeast is unicellular and can be grown as a haploid, it is very easy to isolate mutants. Genetic variation can be further extended by mating of haploid strains. It should be mentioned that $S$. cerevisiae is the first eukaryotic organism whose genome has been completely sequenced. Approximately 6000 genes have been identified and the information on that is freely available via Internet at http://www. yeastgenome.org. However, the functions of many genes are still unknown.

\section{OXIDATIVE STRESS CONCEPT}

In this article we will focus on oxidative stress, accompanying, in fact, any kind of stresses - like heat shock, low temperature exposure, osmotic, xenobiotic stresses, etc. In living cells oxygen molecule can be reduced via a four-electron scheme to produce water molecules as it takes place at the terminal part of the electron transport chain in the reaction catalyzed by cytochrome oxidase. That is a common way for most organisms and it uses over $90 \%$ of oxygen consumed by the cell. Alternatively, the oxygen molecule can be reduced step-by-step via a one-electron way with the formation of partially reduced products such as superoxide anion $\left(\mathrm{O}_{2}^{-\bullet}\right)$,

\footnotetext{
`Part of this article was presented at the Winter School of Biotechnology (Jagiellonian University, Kraków, Poland, 2006) Abbreviations: DDC, N,N'-diethyldithiocarbamate; G6PDH, glucose-6-phosphate dehydrogenase; HSP, heat shock protein; $\mathrm{O}_{2}^{-\bullet}$, superoxide anion; ${ }^{\bullet} \mathrm{OH}$, hydroxyl radical; SOD, superoxide dismutase; TBARS, thiobarbituric acid-reactive substances; ROS, reactive oxygen species.
} 
hydrogen peroxide $\left(\mathrm{H}_{2} \mathrm{O}_{2}\right)$ and hydroxyl radical $\left({ }^{\circ} \mathrm{OH}\right)$, collectively named reactive oxygen species - ROS (Halliwell \& Gutteridge, 1985; Lushchak, 2001; 2002). They can interact with most cellular components resulting in their modification. Therefore, cells possess a complex system neutralizing the deleterious effects of ROS. The most critical component of the protective system consists of so-called antioxidant enzymes. Superoxide dismutase is one of them, which facilitates reduction of $\mathrm{O}_{2}^{-}$to $\mathrm{H}_{2} \mathrm{O}_{2}$. The second group includes different peroxidases, particularly catalases. It should be also mentioned that antioxidant enzymes operate in concert with low-molecular mass antioxidants, namely vitamins A, C, E, melanin, uric acid etc. The processes of ROS production and degradation are well balanced in a normally operating cell, but sometimes this balance is disturbed and the situation when the steady-state ROS concentration is enhanced has been called oxidative stress (Halliwell \& Gutteridge, 1985; Lushchak, 2001; 2002). As it was mentioned above, oxidative stress accompanies in fact all known kinds of cell stresses.

\section{FREE RADICAL PROTEIN OXIDATION}

Here we will mainly discuss protein targets for oxidative modification caused by ROS. The set of products of this process depends on many circumstances like ROS nature, medium composition and certainly on the type of protein (Stadtman, 1993; Stadtman \& Levine, 2000). Sulfur-containing amino acids, cysteine and methionine, heterocyclic histidine and tryptophan as well as tyrosine and phenylalanine are the most susceptible to oxidation. A large array of products has been identified and may be potentially used to study the interactions between ROS and proteins. Free radical oxidation of several amino acids, namely cysteine, histidine and some others, results in the formation of additional carbonyl groups in proteins. Due to many reasons, carbonyl groups are used most commonly to evaluate the intensity of ROS attack on proteins (Stadtman \& Levine, 2000). There are several specific approaches, but all of them are based on the interaction of dinitrophenylhydrazine with protein carbonyls resulting in dinitrophenylhydrazones. Further, they can be either determined directly by spectrophotometry or with antibodies developed against dinitrophenylhydrazone protein derivatives (Lenz et al., 1989). Anti2,4-dinitrophenylhydrazone antibodies are produced commercially. The latter technique may be used to quantify protein carbonyls in vitro or detect them in tissues via cell staining in situ. Certainly, it is clear that there is always a background level of protein carbonyls and all measured changes should be com- pared with "control" level. Recently new techniques to measure concentrations of oxidatively modified proteins have been developed. Although not used extensively in yeast yet, they have a great potential in this area as well. One of them includes the use of biotin-hydrazide and avidin-fluorescein affinity staining for labelling and a two-dimensional electrophoresis combined with matrix-assisted laser desorption/ionization mass spectroscopy - MALDIMS (Yoo \& Regnier, 2004). Another related example is the use of Girard P reagent and identification of oxidation sites using MALDI-TOF analysis (Mirzaei \& Regnier, 2006).

\section{YEAST AS A MODEL}

To our knowledge, the use of antibodies to identify specific target proteins oxidized in yeast was first proposed by Joaquim Ros and colleagues (Cabiscol et al., 2000). They found that in aerobically respiring S. cerevisiae cells hydrogen peroxide and menadione induced oxidation of mitochondrial proteins such as E2 subunits of both pyruvate dehydrogenase and $\alpha$-ketoglutarate dehydrogenase, aconitase, heat shock protein 60 , and $\alpha$-subunit of cytosolic fatty acid synthase and glyceraldehyde-3-phosphate dehydrogenase. Under fermentative conditions hydrogen peroxide and menadione induced oxidation of pyruvate decarboxylase, enolase, fatty acid synthase and glyceraldehyde-3-phosphate dehydrogenase (Cabiscol et al., 2000). Simultaneously, the authors found that the oxidants listed above reduced the activity of fatty acid synthase, aconitase, pyruvate decarboxylase, pyruvate dehydrogenase, $\alpha$-ketoglutarate dehydrogenase, enolase and glyceraldehyde-3-phosphate dehydrogenase. Therefore, one can conclude that in vivo inactivation of enzymes via oxidation is accompanied by the accumulation of carbonyl groups in respective proteins. When yeast cells were stressed by oxidants, fermenting cells exhibited lower viability, increased peroxide generation as well as higher concentrations of protein carbonyls and lipid peroxides than aerobically respiring ones (Cabiscol et al., 2000).

The use of two-dimensional gel electrophoresis and Western blot analysis combined with fingerprint mass spectrometry allowed the identification of specific oxidatively modified proteins in replicatively and chronologically aged S. cerevisiae cultures (Reverter-Branchat et al., 2004). Although in both aging models the metabolic peculiarities were different, the major targets were almost the same. Common targets included stress resistance proteins, HSP60 and HSP70, and enzymes involved in glucose metabolism such as enolase, glyceraldehyde-3-phosphate dehydrogenase, fructose-1,6-bisphosphate aldolase, 
pyruvate decarboxylase, and alcohol dehydrogenase. It is interesting to note that caloric restriction reduced the levels of products of free radical oxidation of proteins and lipids (Reverter-Branchat et al., 2004).

In 1999 Spanish researchers demonstrated that glutaredoxin Grx5 localized in mitochondria protected S. cerevisiae cells against oxidative damage caused by menadione and hydrogen peroxide (RodríguezManzaneque et al., 1999). Deletion of the gene coding for Grx5 significantly increased the levels of protein carbonyls; besides, several specific proteins, including transketolase, appeared to be highly sensitive to oxidation. This work was further developed and it was found that Grx5 also protected enzymes containing Fe/S-clusters (Rodríguez-Manzaneque et al., 2002). The enzyme Grx5 may be involved either in the synthesis of $\mathrm{Fe} / \mathrm{S}$-clusters or in maintaining their native structure. Anyway, a $\Delta g r x 5$ strain demonstrated also increased intracellular iron levels (Rodríguez-Manzaneque et al., 2002).

The role of another mitochondrial stress protein, the molecular chaperone HSP60, in protection of $S$. cerevisiae against oxidative stress was also studied (Cabiscol et al., 2002). This protein is an essential mitochondrial chaperone which promotes the folding of many proteins imported into the mitochondrial matrix as well as directs several proteins into the intermembrane space. Protection against protein carbonylation and of Fe/S-containing enzymes from oxidative inactivation was found to be a dose-dependent with respect to HSP60 levels. The concentrations of intracellular iron labile pool also positively correlated with HSP60 levels. The authors concluded that the chaperone defended yeast cells protecting several $\mathrm{Fe} / \mathrm{S}$-proteins against oxidation and in turn preventing the release of iron ions (Cabiscol et al., 2002). Poyton and colleagues studied a possible protective role of both yeast superoxide dismutases (SODs) - Cu,Zn-SOD, encoded by the SOD1 gene and localized in both the cytosol and the mitochondrial intermembrane space, and Mn-SOD, encoded by the SOD2 gene and localized within mitochondrial matrix (O'Brien et al., 2004). Using protein carbonylation as a measure of oxidative stress intensity, no correlation was found between the overall levels of respiration and the concentration of oxidatively damaged mitochondrial proteins in parental either single or double SOD mutants. Moreover, mitochondrial protein carbonylation levels in the single or double mutants were the same regardless whether the cells were collected at the mid-logarithmic or early-stationary phases, demonstrating that the two SODs are not required for protection of the majority of mitochondrial proteins from oxidative damage under these conditions ( $\mathrm{O}^{\prime}$ Brien et al., 2004). However, during late stationary phase, the levels of carbonylated mitochondrial proteins increased in all strains, especially in the sod1 and sod1sod2 mutants. Among the oxidized proteins in the SOD mutants, at least several should be mentioned. These are cytosolic glyceraldehyde-3-phosphate dehydrogenase and enolase, and mitochondrial $\alpha$-chain of ATP synthase, aconitase, subunit 2 of isocitrate dehydrogenase, etc. Some of these proteins are carbonylated in both mutants, but others are not. Therefore, the authors concluded that $\mathrm{Mn}-\mathrm{SOD}$ and $\mathrm{Cu}, \mathrm{Zn}-\mathrm{SOD}$ have both unique and overlapping functions ( $\mathrm{O}^{\prime}$ Brien et al., 2004).

It is clear from the works analyzed above and some others that the identification of protein carbonyl levels as a marker of oxidative damage of proteins has both advantages and disadvantages. Certainly, the techniques based on this principle are most commonly used because they are well developed, reproducible, available and cheap. However, there are many other products of protein oxidation by ROS which are not determined with dinitrophenylhydrazine. They are missed and that is why false conclusions may be drawn. Separation of proteins by gel electrophoresis for further identification with respective antibodies also may result in reduced sensitivity (Rodney Levine, personal communication). Therefore, investigations in our laboratory have been concentrated on the role of antioxidant enzymes in the protection of cellular components from oxidative damage with the focus on proteins with enzymatic activity. We did evaluate concentrations of protein carbonyls, but mainly paid attention to the activity of enzymes known to be inactivated by ROS. The investigations were strengthened by correlative analysis, giving a mathematical description of respective relationships. Several approaches were used to modify activities of antioxidant enzymes: (i) the use of respective single or double mutants; (ii) the use of certain specific conditions of yeast cultivation, and finally (iii) the inhibition of antioxidant enzymes.

A significant correlation was found between the markers of oxidative damage of lipids, so-called thiobarbituric acid-reactive substances - TBARS, and protein carbonyls when parental and catalasedeficient yeast strains were grown on glucose, a fermentative substrate, to the early stationary phase (Lushchak \& Gospodaryov, 2005). The strong positive correlation (Fig. 1) may suggest an involvement of lipid peroxidation products in the formation of additional carbonyl groups in proteins (Halliwell \& Gutteridge, 1985; Stadtman, 1993; Stadtman \& Levine, 2000). When the same set of strains was grown on ethanol, an oxidative substrate, the correlation mentioned above was not found. However, other relationships were disclosed. Figure 2A demonstrates a strong negative linear correlation between catalase activities and levels of protein carbonyls. When glu- 


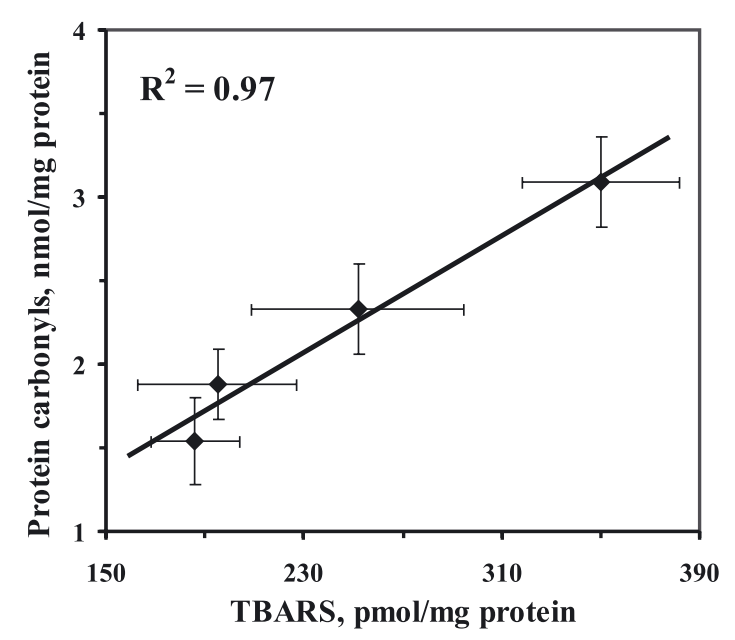

Figure 1. The relationship between protein carbonyl levels and thiobarbituric-reactive substances (TBARS) content in wild-type and catalase-deficient $S$. cerevisiae strains grown on glucose.

From Lushchak and Gospodaryov (2005), with permission.

cose-6-phosphate dehydrogenase (G6PDH) activities were plotted against catalase ones, a very strong positive correlation was found (Fig. 2B). Both findings may suggest that catalase might be responsible for the protection of cellular proteins against oxidation by ROS and that G6PDH may be one of the favorable oxidized targets under conditions used. Our conclusion on the possible involvement of ROS in G6PDH inactivation was supported by in vitro experiments, where partially purified enzyme was subjected to ROS attack (Gospodaryov et al., 2005). It may be added that when catalase was inhibited in vivo by 3-amino-2,4-triazole, similar correlation between catalase activities and protein carbonyl levels was found in goldfish brain (Bagnyukova et al., 2005).

To study the role of superoxide dismutases in the protection of yeast cell proteins from ROS-induced damage, four yeast strains were used: parental, defective in SOD1, in SOD2 and in both (Lushchak et al., 2005a). In these experiments, yeast cells were grown on the oxidative substrate glycerol. As it could be expected, wild-type yeast demonstrated the maximal SOD activity followed by $\Delta s o d 2$ and $\Delta$ sod1, but the double mutant surprisingly also had a small, but reproducible activity. It could be removed by dialysis and was not visible at native gel electrophoresis of cellular proteins developed for SOD activity. When protein carbonyl levels were plotted against SOD activities, a nonlinear relationship was found, which could be better described by a parabola (Fig. 3). A similar relationship between SOD activity and lipid peroxidation in vitro was described by Joy McCord (1997). Further, we used another approach and for this reason the Mn-SOD-defective strain was chosen (Lushchak et al., 2005b). It pos-
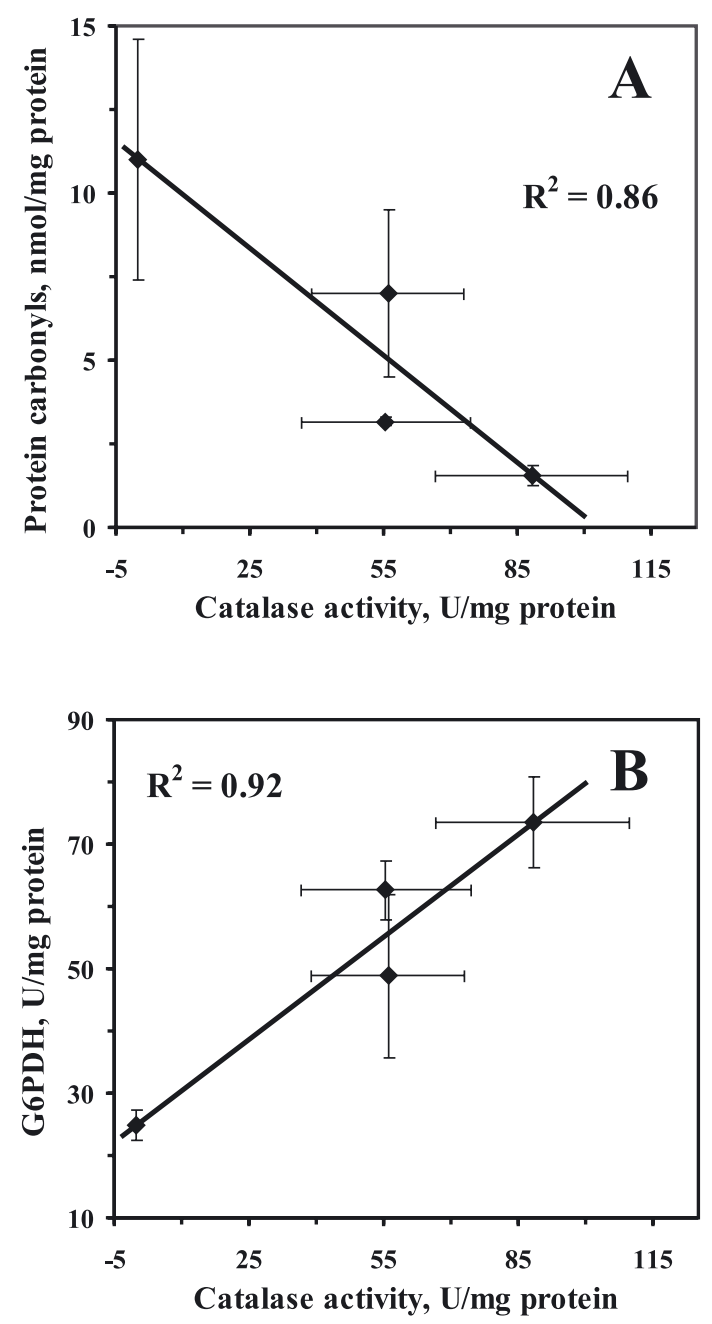

Figure 2. The relationship between catalase activities and protein carbonyl levels (A) and activities of catalase and glucose-6-phosphate dehydrogenase (B) in wild-type and catalase-deficient $S$. cerevisiae strains grown on ethanol. From Lushchak and Gospodaryov (2005), with permission.

sesses only $\mathrm{Cu}, \mathrm{Zn}$-SOD which may be inhibited by $\mathrm{N}, \mathrm{N}^{\prime}$-diethyldithiocarbamate (DDC). This compound inhibits $\mathrm{Cu}, \mathrm{Zn}$-SOD activity by chelating and removing $\mathrm{Cu}$ (II) ions from the enzyme active site (Cocco et al., 1981; Benov \& Fridovich, 1996). This made it possible to get patterns with different $\mathrm{Cu}, \mathrm{Zn}-\mathrm{SOD}$ activities. Surprisingly, protein carbonyl levels and SOD activities demonstrated a bell-shaped curve with a maximal SOD activity of $150 \mathrm{U} / \mathrm{mg}$ protein (Fig. 4). It seems that lower and higher SOD activities had better protective effects against protein oxidation. It should be noted that all the effects of DDC in the strain possessing $\mathrm{Cu}, \mathrm{Zn}$-SOD were not found in the SOD-null mutant. That is why we concluded that the modification of protein carbonyl levels was a result of $\mathrm{Cu}, \mathrm{Zn}-\mathrm{SOD}$ inhibition (Lushchak et al., 2005b).

Several interesting facts were found by studying the activities of enzymes in yeast strains possess- 


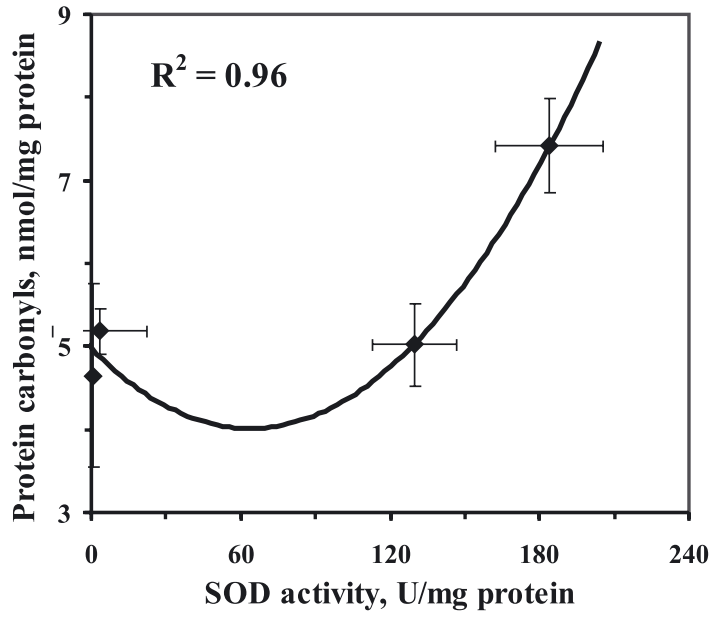

Figure 3. The relationship between SOD activities and carbonyl protein levels in wild-type and SOD-deficient S. cerevisiae strains.

From Lushchak et al. (2005a), with permission.

ing different SOD activities (Lushchak et al., 2005a; 2005b). First, catalase and superoxide dismutase activities were positively and linearly related to each other with a high correlation coefficient. This was observed by both approaches, with the use of a set of SOD-defective mutants (Fig. 5A) and with the inhibition of $\mathrm{Cu}, \mathrm{Zn}$-SOD by DDC (Fig. 5B) (Lushchak et al., 2005a; 2005b). Similar relationships were found between SOD and catalase activities when yeast cells were treated with hydrogen peroxide (Bayliak et al., 2006). Therefore, one may suggest that in vivo SOD and catalase activities depend on each another. Second, the strong negative linear correlation between SOD and G6PDH activities (Lushchak et al., 2005b) demonstrates that not superoxide anion, but rather

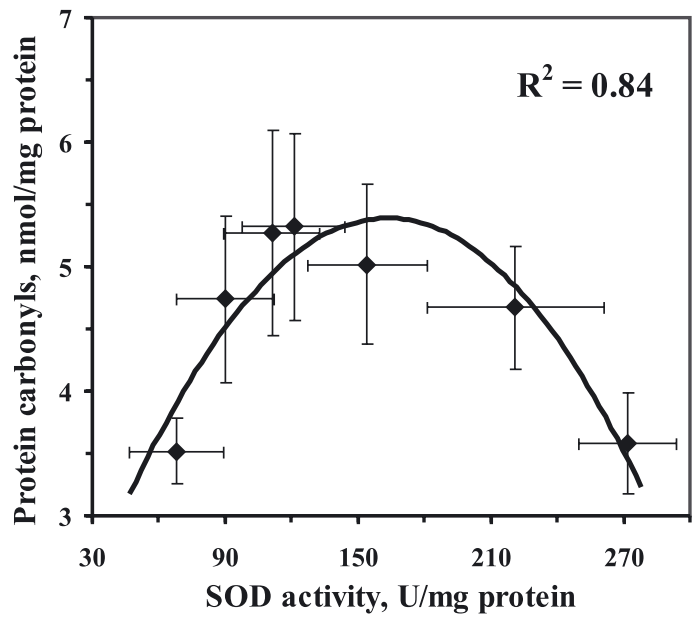

Figure 4. The relationship between SOD activities and carbonyl protein levels in Mn-SOD-deficient S. cerevisiae cells treated with different diethyldithiocarbamate concentrations.

From Lushchak et al. (2005b), with permission.
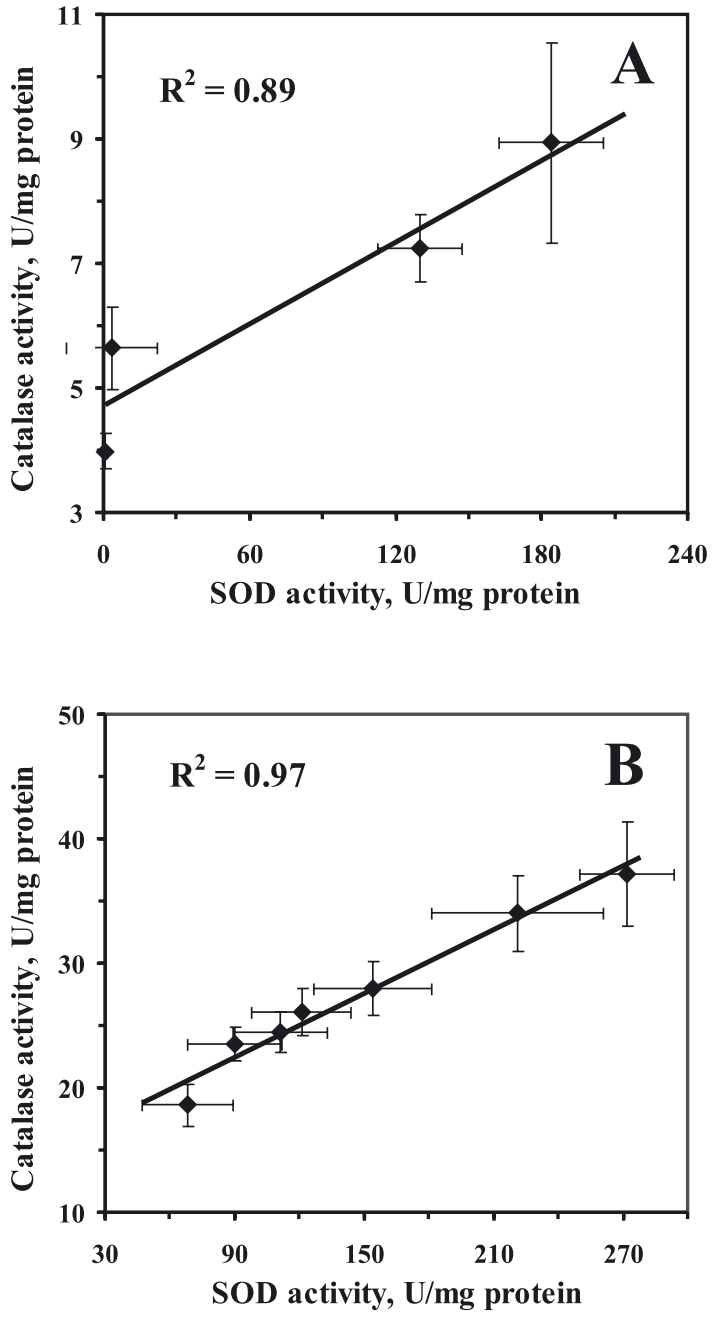

Figure 5. The relationship between activities of SOD and catalase.

A, wild-type; B, SOD-deficient S. cerevisiae strains. From Lushchak et al. (2005a), with permission.

hydrogen peroxide or products of its metabolism might play a critical role in G6PDH inactivation. Finally, a strong linear positive correlation was found between SOD activities, on one hand, and the activities of malate dehydrogenase, on the other. It can be concluded that both SODs and catalases operate in concert to protect cellular proteins from oxidation by ROS, but they may act in different ways because they reduce the cellular levels of superoxide anion and hydrogen peroxide, respectively.

Generally, it is clear that being unicellular and well studied with well developed genetic and biochemical set of manipulations baker's yeast is an excellent model for many sorts of experiments. Here we have demonstrated only one example where yeast was used as a model to study the interaction between ROS and cellular components with particular interest in protein oxidation. The possibility of easy manipulation of antioxidant enzymes allow deciphering their role in situ and the broad homolo- 
gies between yeast and metazoan genomes transfer of knowledge and in some cases even understanding of the nature of human and animal diseases.

\section{Acknowledgements}

This research received partial support by a grant of the Ministry of Education and Science of Ukraine (\#0102U003439). I thank Dr. T. Bagnyukova for the critical reading of the manuscript and an anonymous referee for useful comments and recommendations which helped to improve the manuscript.

\section{REFERENCES}

Bagnyukova T, Vasylkiv O, Storey K, Lushchak V (2005) Catalase inhibition by amino triazole induces oxidative stress in goldfish brain. Brain Res 1052: 180-186.

Bayliak MM, Semchyshyn HM, Lushchak VI (2006) Effect of hydrogen peroxide on antioxidant enzyme activities in Saccharomyces cerevisiae is strain-specific. Biochemistry (Moscow) 71: 1243-1252.

Benov L, Fridovich I (1996) Functional significance of the $\mathrm{Cu}, \mathrm{ZnSOD}$ in Escherichia coli. Arch Biochem Biophys 327: 249-253.

Cabiscol E, Piulats E, Echave P, Herrero E, Ros J (2000) Oxidative stress promotes specific protein damage in Saccharomyces cerevisiae. J Biol Chem 275: 27393-27398.

Cabiscol E, Belli G, Tamarit J, Echave P, Herrero E, Ros J (2002) Mitochondrial Hsp60, resistance to oxidative stress, and the labile iron pool are closely connected in Saccharomyces cerevisiae. J Biol Chem 277: 44531-44538.

Cocco D, Calabrese L, Rigo A, Argese E, Rotillio G (1981) Re-examination of the reaction of diethyldithiocarbamate with the copper of superoxide dismutase. J Biol Chem 256: 8983-8986.

Gospodaryov D, Bayliak M, Lushchak V (2005) Free radical in vitro inactivation of glucose-6-phosphate dehydrogenase from the yeast Saccharomyces cerevisiae. Ukrainian Biochem J 77: 54-60.

Halliwell B, Gutteridge JMC (1985) Free Radicals in Biology and Medicine. Clarendon, Oxford.

Lenz A, Costabel U, Shaltiel S, Levine R (1989) Determination of carbonyl groups in oxidatively modified proteins by reduction with tritiated sodium borohydride. Anal Biochem 177: 419-425.

Lushchak VI (2001) Oxidative stress and mechanisms of protection against it in bacteria. Biochemistry (Moscow) 66: $476-489$.
Lushchak VI (2002) Oxidative stress in bacteria. In Oxidative Stress at Molecular, Cellular and Organ Levels (Johnston P, Boldyrev AA eds) pp 45-65. Research Singpost, Trivandrum, India.

Lushchak V, Gospodaryov D (2005) Catalases protect cellular proteins from oxidative modification in Saccharomyces cerevisiae. Cell Biol Intern 29: 187-192.

Lushchak V, Semchyshyn H, Mandryk S, Lushchak O (2005a) Possible role of superoxide dismutases in the yeast Saccharomyces cerevisiae under respiratory conditions. Arch Biochem Biophys 441: 35-40.

Lushchak V, Semchyshyn H, Lushchak O, Mandryk S (2005b) Diethyldithiocarbamate inhibits in vivo Cu,Znsuperoxide dismutase and perturbs free radical processes in yeast Saccharomyces cerevisiae. Biochem Biophys Res Commun 338: 1739-1744.

McCord J (1997) The importance of oxidant-antioxidant balance. In Oxidative Stress, Cancer, AIDS, and Neurodegenerative Diseases (Montagnier L, Olivier R, Pasquier C eds) pp 1-7. Marcel Dekker, New York.

Mirzaei H, Regnier F (2006) Affinity chromatographic selection of carbonylated proteins followed by identification of oxidation sites using tandem mass spectrophotometry. Anal Chem 78: 770-778.

O'Brien K, Dirmeier R, Engle M, Poyton R (2004) Mitochondrial protein oxidation in yeast mutants lacking manganese-(MnSOD) or copper- and zinc-containing superoxide dismutase (CuZnSOD): evidence that MnSOD and CuZnSOD have both unique and overlapping functions in protecting mitochondrial proteins from oxidative damage. J Biol Chem 279: 51817-51827.

Reverter-Branchat G, Cabiscol E, Tamarit J, Ros J (2004) Oxidative damage to specific proteins in replicative and chronological-aged Sacharomyces cerevisiae: common targets and prevention by calorie restriction. J Biol Chem 279: 31983-31989.

Rodríguez-Manzaneque MT, Ros J, Cabiscol E, Sorribas A, Herrero E (1999) Grx5 glutaredoxin plays a central role in protection against protein oxidative damage in Saccharomyces cerevisiae. Mol Cell Biol 19: 8180-8190.

Rodríguez-Manzaneque MT, Tamarit J, Belli G, Ros J, Herrero E (2002) Grx5 is a mitochondrial glutaredoxin required for the activity of iron/sulfur enzymes. Mol Biol Cell 13: 1109-1121.

Stadtman E (1993) Oxidation of free amino acid residues in proteins by radiolysis and by metal-catalyzed reactions. Annu Rev Biochem 62: 797-821.

Stadtman E, Levine R (2000) Protein oxidation. Ann NY Acad Sci USA 899: 191-208.

Yoo BS, Regnier FE (2004) Enrichment of carbonylated peptides using Girard P reagent and strong cation exchange chromatography. Electrophoresis 25: 1334-1341. 